\title{
Performance of New Sugarcane Varieties in the Northern Humid Coastal Plains of Puerto Rico ${ }^{1}$
}

\author{
Rafael Gandía Caro, Carlos González Molina, and \\ Edwin Acevedo Borrero²
}

\begin{abstract}
Three sites were selected in the northern humid coastal plains of Puerto Rico for the performance evaluation of 57 new sugarcane varieties. The experiments were located on typical soils of the sugarcane producing northern coastal plain region. A plant crop (fall planting) and two ratoons were harvested at each site.

On the San Francisco farm between Arecibo and Utuado, the outstanding new varieties which outyielded all the others tested were PR 64-2548, PR 1152, PR 65-325 and PR 64-2705. Of these, PR 1152 and PR 64-2705 are the most promising varieties in this area because of their erect growth and the good quality of their juice.

On the Las Claras farm, near Arecibo, the most promising new varieties for the area were PR 1141 and PR 62-258.

On the Consejo farm at Barrio Bajadero, Arecíbo, varieties PR 1117, PR 1152, Selección Sóller, PR 1048, PR 62-469 and the PR 65-2523 performed best on the basis of cane tonnage. As a commercial variety for the area, PR 1152 was the most promising variety, having a good juice quality and intermediate cane tonnage per acre ( 40 to 45 tons). It can be harvested mechanically without difficulties because of its erect growth habit.

PR 980 produced good tonnage, but was low in sucrose content, indicating that it should not be recommended for the northern humid coastal plains of Puerto Rico.
\end{abstract}

\section{INTRODUCTION}

The farm value of the sugarcane crop in Puerto Rico in 1973-74 was $\$ 70,945,000$ (2). Crop yield and acreage have decreased sharply during the past 15 years. About 263,347 acres were planted to sugarcane in 1966-67; only 140,000 in 1973-74. Mean cane yield in 1974 was

${ }^{1}$ Manuscript submitted to Editorial Board March 10, 1975.

${ }^{2}$ Associate Agronomist, Plant Breeder and Research Assistant, respectively, Agricultural Experiment Station, Mayagüez Campus, University of Puerto Rico, Río Piedras, Puerto Rico. 
about 30 tons/acre with an $8.013 \%$ sucrose content for a total sugar production of 287,269 tons (4).

Sugarcane hybridization, selection for disease resistance, and evaluation are the three phases of the Experiment Station's sugarcane variety research program. The goal is to develop varieties that outyield those in present commercial use and also are suitable for mechanized cultivation and harvesting. One of the variety testing areas is located in the northern humid coastal plains in the vicinity of Cambalache sugar mill. In 1974 there were approximately 15,550 acres planted to sugarcane in this region. A total of 462,752 tons of cane were ground with a mean sucrose content of $7.52 \%$. The area's principal varieties are PR 1028 (early maturing, high sucrose), H $32-8560$ (intermediate maturing, good juice quality), and PR 980 (late maturing cane with low juice quality, particularly on poorly drained soils). A large proportion of the area's cane is harvested mechanically. Much damaged cane is left in the field after harvesting, and the cane arriving at the mill is high in foreign matter and low in sugar content. Previous work conducted in 1967-68 showed that varieties PR 62-626, PR 62-357 and PR 62-147 produced 21, 10 and $7 \%$ more sugar, respectively, than the standard variety PR 980 in that area (1). None, however, outyielded PR 1028.

This paper reports the results from field experiments conducted from 1970 to 1974 at three sites in the region, involving 57 varieties selected from the 1961-65 breeding program together with two standard commercial varieties.

Several features must be considered in addition to yield performance in a program geared toward replacement of traditional varieties by new ones in attempts to meet industry requirements in a particular area. Varieties should have high juice quality, and produce a relatively high tonnage. Further, they must be suitable for mechanized harvesting. Lodged or badly inclined canes are unsuitable for harvesting with machines designed for erect or semi-erect stalks. Propensity to lodge is thus a decisive feature which normally disqualifies a candidate variety.

Special consideration also should be given to maturity. A planned

TABLE 1.-Rainfall data (inches) for each crop season at the three experimental sites

\begin{tabular}{lrcc}
\hline \multirow{2}{*}{ Site } & \multicolumn{3}{c}{ Rainfall } \\
\cline { 2 - 4 } & Plant cane & First ratoon & Second ratoon \\
\hline San Francisco & 102.5 & 66.8 & 52.7 \\
Las Claras & 97.9 & 66.6 & 55.3 \\
Consejo & 107.9 & 72.5 & 56.3 \\
\hline
\end{tabular}


balance of early-to-late maturity varieties provides full-grown or fully ripe cane throughout the harvest season. Agronomic characteristics such as germination, growth habit, stooling, stalk development, lodging and ratooning also should receive due consideration.

Fiber content is of considerable importance. An accepted fiber range is from 12 to $16 \%$. Fiber over $16 \%$ is considered undesirable from the standpoint of juice extraction in the factory. Varieties with less than $12 \%$ may exhibit recumbent growth in the humid northern coastal plains even though they are low-tonnage canes.

\section{MATERIALS AND METHODS}

The experiments were located as follows: San Francisco farm between Arecibo and Utuado; Las Claras farm near Cambalache Sugar Mill, and Consejo farm at Barrio Bajadero, Arecibo.

The soil on the San Francisco farm is classified as Coloso, an Aeric Tropic Fluvaquents, fine, mixed, non-acid, isohyperthermic (3). Thirty varieties were tested in a $5 \times 6$ rectangular lattice design experiment, with six replications. The experiment was planted in October 1970 (fall planting) and harvested in February 1972 at 16 months of age. The first ratoon crop was harvested in April 1973 at $13^{1 / 2}$ months while the second was harvested in April 1974 at 12 months.

On the Las Claras farm, the soil is classified as Sabana Seca, an Oxic Plinthaquults, clayey, mixed, isohyperthermic (3). Thirty varieties were tested in a $5 \times 6$ balanced lattice design experiment with six replications. The experiment was planted in October 1970 (fall planting) and harvested in February 1972 at $16^{1 / 2}$ months of age. The first ratoon crop was harvested in April 1973 at $13 \frac{1}{2} 2$ months while the second was harvested in April 1974 at 12 months.

On the Consejo farm, the soil is classified as Toa, a Fluventic Hapludolls, fine, mixed, isohyperthermic (3). Twenty-eight varieties were compared in a partially balanced incomplete block design experiment with six replications. The experiment was planted in October 1970 (fall planting) and harvested in March 1972 at 16 months of age. The first ratoon crop was harvested in April 1973 at 131.2 months while the second was harvested in April 1974 at 12 months.

The plots were $22 \times 20 \mathrm{ft}$ with furrows at $5^{1 / 2} \mathrm{ft}$ at all sites. Cultivation, fertilization, weeding and crop protection followed standard Puerto Rican practices for each location. Observations were conducted periodically relative to germination, stooling, growth habit, stalk characteristics (vigor, thickness, height, solidity and lodging), ripening, ratooning and others. Rainfall data was recorded at each site (table 1). The cane was burned prior to harvesting and then cut with machetes. Data was 
TABLE 2.-Means yields and relative values of 30 sugarcane varieties in the experimental field at San Francisco Farm

\begin{tabular}{|c|c|c|c|c|c|c|c|c|}
\hline \multirow[t]{2}{*}{ Rank } & \multirow[t]{2}{*}{ Variety } & \multirow[t]{2}{*}{$\begin{array}{l}\text { Sucrose } \\
\text { content }\end{array}$} & \multirow[t]{2}{*}{$\mathrm{TCA}^{\mathrm{A}}$} & \multirow[t]{2}{*}{$\mathrm{TSA}^{2}$} & \multicolumn{2}{|c|}{$\begin{array}{l}\text { Relative value on TSA as } \\
\text { compared to checks }\end{array}$} & \multirow[t]{2}{*}{$\begin{array}{l}\text { Fiber } \\
\text { content }\end{array}$} & \multirow{2}{*}{$\begin{array}{c}\text { Outyielded } \\
\text { varieties } \\
\text { in TSA } \\
(\mathrm{P}=0.05)\end{array}$} \\
\hline & & & & & PR 980 & PR1028 & & \\
\hline & & $\%$ & & & & & $\%$ & \\
\hline \multicolumn{9}{|c|}{ A. Plant cane crop 1972} \\
\hline 1 & PR $65-325$ & 12.25 & 52.6 & 6.40 & 1.231 & 1.286 & 13.84 & 17 to 30 \\
\hline 2 & PR 64-2548 & 11.84 & 52.5 & 6.16 & 1.184 & 1.239 & 13.19 & 20 to 30 \\
\hline 3 & P R 64-2705 & 13.95 & 43.9 & 6.09 & 1.170 & 1.225 & 13.21 & 21 to 30 \\
\hline 4 & PR 1242 & 11.66 & 50.8 & 5.99 & 1.152 & 1.204 & 14.58 & 23 to 30 \\
\hline 5 & P R 65-292 & 10.95 & 50.1 & 5.56 & 1.067 & 1.117 & 13.10 & 29 to 30 \\
\hline 6 & P R 61-324 & 12.37 & 43.3 & 5.39 & 1.036 & 1.085 & 11.41 & 30 \\
\hline 7 & PR 1152 & 11.93 & 43.3 & 5.27 & 1.011 & 1.058 & 13.77 & 30 \\
\hline 8 & PR 980 & 11.35 & 45.8 & 5.20 & 1.000 & 1.046 & 13.29 & 30 \\
\hline 9 & PR 63-525 & 11.26 & 45.5 & 5.13 & .986 & 1.032 & 12.79 & 30 \\
\hline 10 & P R 61-902 & 12.69 & 40.6 & 5.11 & .982 & 1.027 & 13.82 & 30 \\
\hline 11 & PR 62-456 & 11.33 & 45.5 & 5.09 & .977 & 1.022 & 12.29 & \\
\hline 12 & PR 1028 & 12.21 & 40.7 & 4.97 & .955 & 1.000 & 15.08 & \\
\hline 13 & PR 1140 & 13.71 & 36.5 & 4.95 & .950 & .994 & 13.51 & \\
\hline 14 & P R 65-1539 & 11.28 & 42.7 & 4.91 & .942 & .986 & 14.41 & \\
\hline 15 & PR 64-1791 & 11.26 & 42.1 & 4.74 & .912 & .953 & 14.83 & \\
\hline 16 & PR 1002 & 11.26 & 39.7 & 4.74 & .912 & .951 & 13.27 & \\
\hline 17 & PR 62-285(I) & 12.13 & 37.4 & 4.59 & .882 & .923 & 13.05 & \\
\hline 18 & PR 62-626 & 12.06 & 37.7 & 4.58 & .881 & .922 & 13.58 & \\
\hline 19 & PR 63-227 & 12.00 & 37.6 & 4.52 & .869 & .909 & 12.33 & \\
\hline 20 & PR 63-833 & 12.98 & 35.5 & 4.47 & .858 & .899 & 13.02 & \\
\hline 21 & PR 63-523 & 10.98 & 39.4 & 4.33 & .832 & .871 & 12.13 & \\
\hline 22 & P R 65-229 & 12.08 & 35.3 & 4.33 & .832 & .871 & 13.10 & \\
\hline 23 & PR 1048 & 11.00 & 39.1 & 4.31 & .829 & .867 & 12.52 & \\
\hline
\end{tabular}




\begin{tabular}{|c|c|c|c|c|c|c|c|c|}
\hline 24 & PR 63-861 & 12.17 & 35.9 & 4.31 & .829 & .867 & 12.48 & \\
\hline 25 & PR 62-469 & 11.54 & 36.5 & 4.27 & .821 & .859 & 12.96 & \\
\hline 26 & PR 63-851 & 11.08 & 37.7 & 4.22 & .811 & .849 & 14.74 & \\
\hline 27 & PR 63-489 & 11.76 & 35.0 & 4.15 & .797 & .835 & 11.81 & \\
\hline 28 & PR 63-192 & 11.01 & 38.8 & 4.15 & .797 & .835 & 13.82 & \\
\hline 29 & PR 63-862 & 12.19 & 31.4 & 3.79 & .729 & .763 & 14.68 & \\
\hline 30 & PR 1016 & 12.29 & 27.1 & 3.39 & .651 & .682 & 13.74 & \\
\hline \multicolumn{9}{|c|}{ B. First ratoon crop 1973} \\
\hline 1 & PR 980 & 12.09 & 51.83 & 6.22 & 1.000 & 1.271 & 17.27 & 20 to 30 \\
\hline 2 & PR 1152 & 11.88 & 49.97 & 5.86 & .940 & 1.196 & 15.51 & 23 to 30 \\
\hline 3 & PR 1002 & 12.20 & 47.87 & 5.83 & .935 & 1.190 & 15.63 & 22 to 30 \\
\hline 4 & PR $64-2548$ & 11.90 & 45.90 & 5.40 & .866 & 1.102 & 17.79 & 28 to 30 \\
\hline 5 & PR 62-285(I) & 11.72 & 44.85 & 5.32 & .855 & 1.087 & 16.23 & 29 to 30 \\
\hline 6 & PR 1048 & 12.07 & 44.09 & 5.29 & .850 & 1.081 & 15.64 & 29 to 30 \\
\hline 7 & PR 64-2705 & 12.37 & 42.38 & 5.28 & .848 & 1.079 & 16.24 & 29 to 30 \\
\hline 8 & Pgr 63-192 & 11.85 & 43.83 & 5.27 & .845 & 1.075 & 16.93 & 29 to 30 \\
\hline 9 & P R 62-626 & 12.40 & 40.95 & 5.11 & .821 & 1.044 & 18.25 & 30 \\
\hline 10 & PR 65-325 & 11.71 & 43.44 & 5.05 & .811 & 1.032 & 15.56 & \\
\hline 11 & P R 63-525 & 12.06 & 41.44 & 5.05 & .811 & 1.032 & 15.56 & \\
\hline 12 & P R 62-456 & 12.46 & 39.96 & 5.02 & .805 & 1.024 & 15.65 & \\
\hline 13 & P R 63-523 & 12.04 & 41.91 & 5.02 & .805 & 1.024 & 15.63 & \\
\hline 14 & P R 1028 & 11.44 & 42.84 & 4.89 & .786 & 1.000 & 18.02 & \\
\hline 15 & P R 65-1539 & 13.50 & 36.27 & 4.88 & .782 & .995 & 16.04 & \\
\hline 16 & P R 63-861 & 12.14 & 40.71 & 4.88 & .782 & .995 & 14.59 & \\
\hline 17 & PR 63-851 & 11.85 & 42.51 & 4.88 & .782 & .995 & 17.43 & \\
\hline 18 & PR 61-324 & 14.17 & 32.86 & 4.72 & .759 & .963 & 15.84 & \\
\hline 19 & PR 65-292 & 10.93 & 42.65 & 4.71 & .757 & .961 & 15.87 & \\
\hline 20 & PR 63-227 & 12.51 & 36.37 & 4.58 & .736 & .936 & 16.47 & \\
\hline 21 & PR 1140 & 11.23 & 40.57 & 4.50 & .723 & .920 & 16.28 & \\
\hline
\end{tabular}


Table 2.-Continued

\begin{tabular}{|c|c|c|c|c|c|c|c|c|}
\hline \multirow{2}{*}{ Rank } & \multirow{2}{*}{ Variety } & \multirow{2}{*}{$\begin{array}{l}\text { Sucrose } \\
\text { content }\end{array}$} & \multirow{2}{*}{$\mathrm{TCA}^{1}$} & \multirow{2}{*}{$\mathrm{TSA}^{2}$} & \multicolumn{2}{|c|}{$\begin{array}{l}\text { Relative value on TSA as } \\
\text { compared to checks }\end{array}$} & \multirow{2}{*}{$\begin{array}{l}\text { Fiber } \\
\text { content }\end{array}$} & \multirow{2}{*}{$\begin{array}{c}\text { Outyielded } \\
\text { varieties } \\
\text { in TSA } \\
(\mathrm{P}=0.05)\end{array}$} \\
\hline & & & & & PR 980 & PR1028 & & \\
\hline \multicolumn{7}{|c|}{$\%$} & \multicolumn{2}{|l|}{$\%$} \\
\hline 22 & PR 64-1791 & 9.87 & 43.69 & 4.21 & .676 & .860 & 17.98 & \\
\hline 23 & PR 63-489 & 12.21 & 33.86 & 4.16 & .668 & .850 & 16.23 & \\
\hline 24 & PR 1242 & 10.01 & 41.24 & 4.12 & .660 & .840 & 17.48 & \\
\hline 25 & PR 1016 & 13.70 & 29.48 & 4.02 & .644 & .820 & 16.28 & \\
\hline 26 & PR 62-469 & 11.30 & 34.69 & 3.94 & .633 & .805 & 15.74 & \\
\hline 27 & PR $65-229$ & 11.50 & 32.28 & 3.83 & .614 & .781 & 16.09 & \\
\hline 28 & PR 63-862 & 11.24 & 33.76 & 3.78 & .606 & .770 & 17.29 & \\
\hline 29 & PR 61-902 & 10.85 & 33.41 & 3.61 & .580 & .738 & 17.07 & \\
\hline 30 & PR 63-833 & 12.33 & 29.25 & 3.57 & .573 & .730 & 15.65 & \\
\hline \multicolumn{9}{|c|}{ C. Combined analysis for the plant cane and the first ratoon crop } \\
\hline 1 & PR $64-2548$ & 11.87 & 49.16 & 5.77 & 1.010 & 1.168 & 15.49 & 20 to 30 \\
\hline 2 & PR $65-325$ & 11.99 & 48.22 & 5.75 & 1.007 & 1.164 & 14.89 & 22 to 30 \\
\hline 3 & PR 980 & 11.72 & 48.78 & 5.71 & 1.000 & 1.156 & 15.28 & 23 to 30 \\
\hline 4 & PR 64-2705 & 13.18 & 43.14 & 5.70 & .998 & 1.154 & 14.73 & 23 to 30 \\
\hline 5 & PR 1152 & 11.90 & 46.74 & 5.57 & .977 & 1.129 & 14.64 & 25 to 30 \\
\hline 6 & PR 1002 & 12.05 & 43.73 & 5.26 & .922 & 1.066 & 14.45 & 28 to 30 \\
\hline 7 & PR 65-292 & 10.92 & 46.45 & 5.12 & .896 & 1.036 & 14.49 & 29 to 30 \\
\hline 8 & PR $63-525$ & 11.66 & 43.40 & 5.10 & .894 & 1.034 & 14.18 & 29 to 30 \\
\hline 9 & PR $61-324$ & 13.26 & 38.33 & 5.08 & .889 & 1.028 & 13.63 & 29 to 30 \\
\hline 10 & PR 1242 & 10.83 & 46.24 & 5.07 & .889 & 1.028 & 16.03 & 29 to 30 \\
\hline 11 & PR 62-456 & 11.89 & 42.84 & 5.06 & .885 & 1.024 & 13.97 & 291030 \\
\hline 12 & PR 1028 & 11.82 & 41.84 & 4.94 & .864 & 1.000 & 16.55 & 30 \\
\hline
\end{tabular}




\begin{tabular}{|c|c|c|c|c|c|c|c|c|}
\hline 13 & PR 1048 & 11.53 & 42.59 & 4.93 & .863 & .997 & 14.08 & 30 \\
\hline 14 & PR 62-285(I) & 11.93 & 40.65 & 4.93 & .836 & .997 & 14.64 & \\
\hline 15 & PR 65-1539 & 12.39 & 39.49 & 4.89 & .857 & .991 & 15.23 & \\
\hline 16 & PR 62-626 & 12.23 & 39.30 & 4.84 & .847 & .979 & 15.92 & \\
\hline 17 & PR 11401 & 12.47 & 38.65 & 4.73 & .829 & .959 & 14.90 & \\
\hline 18 & PR 63-523 & 11.51 & 40.61 & 4.67 & .819 & .947 & 13.88 & \\
\hline 19 & PR 63-192 & 11.34 & 40.62 & 4.59 & .805 & .931 & 15.23 & \\
\hline 20 & PR 63-861 & 12.14 & 38.18 & 4.57 & .800 & .925 & 13.54 & \\
\hline 21 & PR 63-227 & 12.26 & 36.96 & 4.57 & .800 & .925 & 14.40 & \\
\hline 22 & PR 63-851 & 11.33 & 40.10 & 4.56 & .798 & .922 & 16.09 & \\
\hline 23 & PR 64-1791 & 10.53 & 42.80 & 4.37 & .766 & .886 & 16.55 & \\
\hline 24 & PR 61-902 & 11.77 & 36.97 & 4.36 & .764 & .884 & 15.45 & \\
\hline 25 & PR 63-489 & 11.99 & 33.95 & 4.11 & .720 & .831 & 14.02 & \\
\hline 26 & PR 65-229 & 11.85 & 33.82 & 4.11 & .720 & .831 & 14.60 & \\
\hline 27 & PR 62-469 & 11.42 & 35.53 & 4.10 & .719 & .830 & 14.35 & \\
\hline 28 & PR 63-833 & 12.66 & 31.89 & 3.97 & .696 & .805 & 14.34 & \\
\hline 29 & PR $63-862$ & 11.74 & 32.71 & 3.80 & .666 & .770 & 15.99 & \\
\hline 30 & PR 1016 & 12.99 & 28.36 & 3.71 & .650 & .752 & 15.01 & \\
\hline
\end{tabular}

${ }^{1} \mathrm{TCA}=$ tons of cane/acre.

${ }^{2}$ TSA $=$ tons of sugar/acre. 
tabulated on cane tonnage per plot and stalk samples were taken for sugar analysis by the pol-ratio method. Tonnage and sugar yield data were evaluated statistically by site and by pool analyses.

\section{RESULTS AND DISCUSSION}

\section{SAN FRANCISCO FARM SITE}

As shown in table $2 \mathrm{~A}$, PR 65-325 was the leading sugar producing variety for a fall plant crop of 16 months of age, at 6.40 tons/acre. PR 64-2548 and PR 64-2705 followed very closely. Details as to the performance of each variety can be obtained from table 2 A. Varieties PR 65-325, PR 2548 and PR 64-2705 produced over 1.2 tons more sugar than the standard commercial varieties of the area, PR 980 and PR 1028. Fiber content of varieties ranged from 11.41 to $15.08 \%$ among the varieties.

The performance of the varieties in the first ratoon followed a different pattern. PR 980 was then the best sugar producer per acre. This is in consequence of the high tonnage of PR 980 coupled with small increases in sucrose content. Fiber content in PR 980 and PR 64-2548 increased to 17.27 and $17.79 \%$ respectively. Yield data for this crop is given in table $2 \mathrm{~B}$.

Due to clarification difficulties with juices, only cane tonnage data is available in the second ratoon. For this reason the combined analysis on plant cane and first ratoon crop presented in table $2 \mathrm{C}$ does not include the second ratoon. The analysis reveals that the most outstanding new varieties were PR 64-2548, PR 65-325, PR 64-2705 and PR 1152. However, considering the juice quality, growth habit and fiber content, PR 1152 and PR 64-2705 appear to be the best adapted to the conditions prevailing at the San Francisco farm.

\section{LAS CLARAS FARM SITE}

Data on the production performance of each variety as a plant crop are given in table $3 \mathrm{~A}$. Variety PR 1141 was the best sugar producer. Mean differences between sugar yield of PR 1141 and all other varieties were significant at the 5\% level except PR 65-325, P.R. 62-285 (I) and H 32 8560. These three varieties were also outstanding and their yields were higher than those of most other varieties including PR 980. Variety PR 1141 produced at least 1 ton/acre more sugar than any other variety; 2 tons/acre more than PR 980. In terms of relative values, the yield increase over variety $\mathrm{H} 32-8560$ was $21 \%$, and over PR 980, $34 \%$.

Yield data for the first ratoon is given in table 3 B. PR 980, which performed poorly in the plant crop, outyielded all other varieties significantly in tons per acre of sugar except PR 62-285 (I), PR 63-525 and PR 62-258. Although PR 980 is also the best sugar producer in the 
TABLE 3.-Mean yields and relative values of 25 sugarcane varieties in the experimental field at Las Claras Farm

\begin{tabular}{|c|c|c|c|c|c|c|c|c|}
\hline \multirow[t]{2}{*}{ Rank } & \multirow{2}{*}{ Variety } & \multirow{2}{*}{$\begin{array}{l}\text { Sucrose } \\
\text { content }\end{array}$} & \multirow{2}{*}{ TCA } & \multirow{2}{*}{ TSA } & \multicolumn{2}{|c|}{$\begin{array}{l}\text { Relative value on TSA as } \\
\text { compared to checks }\end{array}$} & \multirow{2}{*}{$\begin{array}{l}\text { Fiber } \\
\text { content }\end{array}$} & \multirow{2}{*}{$\begin{array}{c}\text { Outyielded } \\
\text { varieties } \\
\text { in TSA } \\
(\mathrm{P}=0.05)\end{array}$} \\
\hline & & & & & H $32-8560$ & PR 980 & & \\
\hline & & $\%$ & & & & & $\%$ & \\
\hline \multicolumn{9}{|c|}{ A. Plant cane crop 1972} \\
\hline 1 & PR 1141 & 11.84 & 66.9 & 7.85 & 1.212 & 1.342 & 13.62 & 5 to 25 \\
\hline 2 & PR $65-325$ & 10.19 & 68.0 & 6.85 & 1.057 & 1.171 & 14.80 & 22 to 25 \\
\hline 3 & PR 62-285(I) & 9.92 & 67.5 & 6.72 & 1.037 & 1.148 & 14.42 & 23 to 25 \\
\hline 4 & $\mathrm{H} \quad 32-8560$ & 10.08 & 65.6 & 6.47 & 1.000 & 1.107 & 12.78 & 23 to 25 \\
\hline 5 & PR 61-53 & 10.94 & 57.6 & 6.36 & .981 & 1.086 & 16.84 & 23 to 25 \\
\hline 6 & PR 1175 & 10.18 & 60.6 & 6.11 & .944 & 1.045 & 13.90 & 24 to 25 \\
\hline 7 & PR $62-521$ & 10.62 & 56.5 & 6.07 & .937 & 1.038 & 14.89 & 24 to 25 \\
\hline 8 & PR 63-489 & 9.73 & 62.2 & 6.06 & .936 & 1.036 & 13.36 & 24 to 25 \\
\hline 9 & PR 62-258 & 9.96 & 60.4 & 6.04 & .933 & 1.033 & 14.00 & 25 \\
\hline 10 & PR 62-626 & 10.94 & 54.7 & 5.92 & .914 & 1.012 & 16.47 & 25 \\
\hline 11 & PR 65-292 & 10.71 & 55.2 & 5.91 & .913 & 1.010 & 13.90 & 25 \\
\hline 12 & PR 980 & 9.63 & 61.4 & 5.85 & .903 & 1.000 & 14.98 & 25 \\
\hline 13 & PR 64-1791 & 9.70 & 60.1 & 5.80 & .896 & .993 & 16.25 & 25 \\
\hline 14 & PR 65-109 & 10.36 & 56.2 & 5.78 & .891 & .987 & 14.33 & 25 \\
\hline 15 & PR $63-227$ & 10.28 & 57.3 & 5.74 & .886 & .981 & 13.86 & 25 \\
\hline 16 & PR $63-525$ & 9.02 & 64.1 & 5.73 & .885 & .980 & 13.98 & 25 \\
\hline 17 & PR 62-739 & 9.20 & 60.0 & 5.47 & .845 & .935 & 14.51 & 25 \\
\hline 18 & PR 63-851 & 9.19 & 58.0 & 5.36 & .828 & .917 & 15.99 & 25 \\
\hline 19 & PR 61-902 & 10.68 & 50.5 & 5.32 & .822 & .910 & 13.34 & 25 \\
\hline 20 & PR 63-488 & 8.62 & 63.1 & 5.27 & .813 & .900 & 12.66 & 25 \\
\hline 21 & PR 1238 & 11.12 & 47.5 & 5.24 & .808 & .895 & 13.16 & 25 \\
\hline 22 & PR 65-229 & 9.94 & 51.6 & 5.16 & .797 & .883 & 15.03 & \\
\hline 23 & PR $65-218$ & 10.43 & 44.7 & 4.70 & .724 & .802 & 15.25 & \\
\hline 24 & PR 62-469 & 9.92 & 45.4 & 4.43 & .684 & .757 & 15.32 & \\
\hline 25 & PR 64-211 & 7.82 & 49.0 & 3.75 & .579 & .641 & 15.07 & \\
\hline
\end{tabular}


Table 3.-Continued

\begin{tabular}{|c|c|c|c|c|c|c|c|c|}
\hline \multirow{2}{*}{ Rank } & \multirow{2}{*}{ Variety } & \multirow{2}{*}{$\begin{array}{l}\text { Sucrose } \\
\text { content }\end{array}$} & \multirow{2}{*}{ TCA } & \multirow{2}{*}{ TSA } & \multicolumn{2}{|c|}{$\begin{array}{l}\text { Relative value on TSA as } \\
\text { compared to checks }\end{array}$} & \multirow{2}{*}{$\begin{array}{l}\text { Fiber } \\
\text { content }\end{array}$} & \multirow{2}{*}{$\begin{array}{c}\text { Outyielded } \\
\text { varieties } \\
\text { in TSA } \\
(\mathrm{P}=0.05)\end{array}$} \\
\hline & & & & & H $32-8560$ & PR 980 & & \\
\hline & & $\%$ & & & & & $\%$ & \\
\hline \multicolumn{9}{|c|}{ B. First ratoon crop 1973} \\
\hline 1 & PR 980 & 12.51 & 51.01 & 6.31 & 1.440 & 1.000 & 17.14 & 5 to 25 \\
\hline 2 & PR 62-285(I) & 12.19 & 47.17 & 5.77 & 1.315 & .912 & 16.91 & 18 to 25 \\
\hline 3 & PR 63-525 & 12.42 & 45.81 & 5.68 & 1.294 & .898 & 14.70 & 18 to 25 \\
\hline 4 & PR 62-258 & 12.31 & 45.38 & 5.61 & 1.280 & .889 & 18.21 & 19 to 25 \\
\hline 5 & PR 1141 & 13.22 & 38.64 & 5.10 & 1.164 & .808 & 14.94 & 24 to 25 \\
\hline 6 & PR 62-626 & 11.26 & 44.51 & 5.05 & 1.152 & .800 & 18.19 & 24 to 25 \\
\hline 7 & PR 65-325 & 12.53 & 40.03 & 5.03 & 1.148 & .797 & 15.44 & 24 to 25 \\
\hline 8 & PR 62-739 & 11.35 & 44.11 & 5.02 & 1.143 & .796 & 16.01 & 24 to 25 \\
\hline 9 & PR 62-469 & 12.84 & 38.70 & 4.94 & 1.127 & .782 & 17.88 & 24 to 25 \\
\hline 10 & PR 63-227 & 12.30 & 40.04 & 4.92 & 1.123 & .779 & 16.39 & 24 to 25 \\
\hline 11 & PR 63-489 & 11.00 & 43.82 & 4.85 & 1.105 & .767 & 16.13 & 24 to 25 \\
\hline 12 & PR 64-1791 & 8.61 & 53.99 & 4.79 & 1.093 & .759 & 18.35 & 24 to 25 \\
\hline 13 & PR 65-109 & 13.12 & 35.96 & 4.77 & 1.086 & .754 & 15.17 & 24 to 25 \\
\hline 14 & PR 61-902 & 11.84 & 40.00 & 4.74 & 1.079 & .749 & 17.91 & 24 to 25 \\
\hline 15 & PR 63-488 & 11.28 & 41.58 & 4.72 & 1.077 & .748 & 14.66 & 24 to 25 \\
\hline 16 & PR 65-292 & 11.88 & 39.76 & 4.70 & 1.073 & .744 & 17.98 & 24 to 25 \\
\hline 17 & PR 62-521 & 11.41 & 40.79 & 4.65 & 1.059 & .735 & 17.63 & 24 to 25 \\
\hline 18 & H $32-8560$ & 11.39 & 38.09 & 4.39 & 1.000 & .694 & 17.22 & \\
\hline 19 & PR 1238 & 12.60 & 33.18 & 4.16 & .949 & .659 & 15.98 & \\
\hline 20 & PR 63-851 & 10.75 & 37.23 & 4.12 & .938 & .651 & 18.85 & \\
\hline 21 & PR 1175 & 12.26 & 33.09 & 4.10 & .933 & .650 & 16.87 & \\
\hline 22 & PR 65-218 & 12.28 & 34.07 & 4.09 & .932 & .648 & 18.19 & \\
\hline 23 & PR 64-211 & 13.45 & 30.32 & 4.08 & .929 & .647 & 17.75 & \\
\hline 24 & PR 65-229 & 11.91 & 28.11 & 3.35 & .762 & .529 & 17.24 & \\
\hline 25 & PR 61-53 & 8.72 & 38.01 & 3.23 & .737 & .511 & 18.38 & \\
\hline
\end{tabular}


Table 3.-Continued

\section{Second ratoon crop 1974}

\begin{tabular}{|c|c|c|c|c|c|c|c|}
\hline PR 980 & 10.27 & 44.08 & 4.51 & 1.288 & 1.000 & 19.27 & 15 to 25 \\
\hline PR 62-258 & 11.19 & 39.68 & 4.41 & 1.259 & .977 & 20.03 & 17 to 25 \\
\hline PR 63-525 & 11.76 & 37.22 & 4.34 & 1.240 & .962 & 16.05 & 18 to 25 \\
\hline PR 65-325 & 12.10 & 35.81 & 4.33 & 1.237 & .960 & 17.98 & 18 to 25 \\
\hline PR 62-739 & 10.86 & 39.44 & 4.31 & 1.231 & .955 & 18.73 & 18 to 25 \\
\hline PR 63-489 & 11.01 & 37.92 & 4.21 & 1.202 & .933 & 16.60 & 18 to 25 \\
\hline PR 1141 & 12.34 & 33.64 & 4.15 & 1.185 & .920 & 17.08 & 18 to 25 \\
\hline PR 62-285(I) & 10.58 & 39.30 & 4.15 & 1.185 & .920 & 17.69 & 18 to 25 \\
\hline PR 62-626 & 10.72 & 38.09 & 4.08 & 1.165 & .904 & 20.02 & 19 to 25 \\
\hline PR 63-227 & 12.40 & 31.78 & 3.92 & 1.120 & .869 & 17.07 & 23 to 25 \\
\hline PR 62-469 & 11.71 & 33.48 & 3.88 & 1.108 & .860 & 17.72 & 24 to 25 \\
\hline PR 63-488 & 10.55 & 36.88 & 3.81 & 1.088 & .844 & 16.33 & 25 \\
\hline PR 64-1791 & 8.90 & 41.15 & 3.71 & 1.059 & .822 & 20.31 & 25 \\
\hline PR 62-521 & 12.03 & 30.13 & 3.67 & 1.048 & .813 & 17.25 & 25 \\
\hline H $32-8560$ & 11.14 & 32.11 & 3.50 & 1.000 & .776 & 17.11 & 25 \\
\hline PR 1175 & 11.68 & 29.58 & 3.49 & .997 & .773 & 17.44 & 25 \\
\hline P R 1238 & 11.34 & 30.01 & 3.41 & .974 & .756 & 16.28 & 25 \\
\hline PR 61-53 & 10.20 & 30.92 & 3.15 & .899 & .698 & 18.62 & 25 \\
\hline PR 61-902 & 10.42 & 30.30 & 3.13 & .894 & .694 & 17.92 & 25 \\
\hline PR 65-292 & 11.38 & 27.69 & 3.10 & .885 & .687 & 17.62 & 25 \\
\hline PR 63-851 & 11.00 & 27.85 & 3.05 & .871 & .676 & 18.98 & 25 \\
\hline PR 64-211 & 10.81 & 27.93 & 3.05 & .871 & .676 & 19.01 & 25 \\
\hline PR $65-109$ & 12.12 & 24.39 & 2.93 & .837 & .649 & 16.16 & \\
\hline PR 65-218 & 10.64 & 27.16 & 2.90 & .828 & .643 & 17.98 & \\
\hline PR $65-229$ & 10.90 & 18.78 & 2.10 & .600 & .465 & 16.93 & \\
\hline \multicolumn{8}{|c|}{ D. Combined analysis } \\
\hline PR 1141 & 12.46 & 46.36 & 5.70 & 1.194 & 1.038 & 15.21 & 10 to 25 \\
\hline PR 62-285(I) & 10.90 & 51.27 & 5.54 & 1.161 & 1.009 & 16.34 & 14 to 25 \\
\hline PR 980 & 10.68 & 52.22 & 5.49 & 1.150 & 1.000 & 17.26 & 14 to 25 \\
\hline PR 65-325 & 11.60 & 47.96 & 5.40 & 1.132 & .983 & 16.07 & 17 to 25 \\
\hline
\end{tabular}


Table 3.-Continued

\begin{tabular}{|c|c|c|c|c|c|c|c|c|}
\hline \multirow{2}{*}{ Rank } & \multirow{2}{*}{ Variety } & \multirow{2}{*}{$\begin{array}{l}\text { Sucrose } \\
\text { content }\end{array}$} & \multirow{2}{*}{$\mathrm{TCA}$} & \multirow{2}{*}{ TSA } & \multicolumn{2}{|c|}{$\begin{array}{l}\text { Relative value on TSA as } \\
\text { compared to checks }\end{array}$} & \multirow{2}{*}{$\begin{array}{l}\text { Fiber } \\
\text { content }\end{array}$} & \multirow{2}{*}{$\begin{array}{c}\text { Outyielded } \\
\text { varieties } \\
\text { in TSA } \\
(\mathrm{P}=0.05)\end{array}$} \\
\hline & & & & & H $32-8560$ & PR 980 & & \\
\hline & & $\%$ & & & & & $\%$ & \\
\hline 5 & PR 62-258 & 11.15 & 48.46 & 5.35 & 1.121 & .974 & 17.41 & 17 to 25 \\
\hline 6 & PR 63-525 & 10.99 & 48.98 & 5.20 & 1.090 & .947 & 14.91 & 20 to 25 \\
\hline 7 & PR 63-489 & 10.60 & 47.96 & 5.05 & 1.058 & .919 & 15.36 & 22 to 25 \\
\hline 8 & PR 62-626 & 11.01 & 45.73 & 5.03 & 1.054 & .916 & 18.23 & 23 to 25 \\
\hline 9 & PR 62-739 & 10.47 & 47.79 & 4.93 & 1.033 & .897 & 16.42 & 23 to 25 \\
\hline 10 & PR 63-227 & 11.65 & 43.01 & 4.85 & 1.016 & .883 & 15.77 & 23 to 25 \\
\hline 11 & PR 62-521 & 11.41 & 42.43 & 4.82 & 1.010 & .877 & 16.59 & 23 to 25 \\
\hline 12 & PR 64-1791 & 9.09 & 51.68 & 4.77 & 1.000 & .868 & 18.30 & 23 to 25 \\
\hline 13 & H $32-8560$ & 10.84 & 45.23 & 4.77 & 1.000 & .868 & 15.70 & 23 to 25 \\
\hline 14 & PR 63-488 & 10.15 & 47.17 & 4.60 & .964 & .837 & 14.55 & 24 to 25 \\
\hline 15 & PR 1175 & 11.40 & 41.06 & 4.58 & .960 & .834 & 16.07 & 24 to 25 \\
\hline 16 & PR 65-292 & 11.32 & 40.85 & 4.56 & .955 & .830 & 16.50 & 24 to 25 \\
\hline 17 & PR 65-109 & 11.86 & 38.83 & 4.49 & .941 & .817 & 15.22 & 24 to 25 \\
\hline 18 & PR 62-469 & 11.52 & 39.14 & 4.43 & .928 & .806 & 16.97 & 25 \\
\hline 19 & PR 61-902 & 11.01 & 40.24 & 4.41 & .924 & .803 & 16.39 & 25 \\
\hline 20 & PR 1238 & 11.68 & 36.87 & 4.26 & .893 & .775 & 15.14 & 25 \\
\hline 21 & PR 61-53 & 9.99 & 42.14 & 4.26 & .893 & .775 & 17.95 & \\
\hline 22 & PR 63-851 & 10.31 & 40.99 & 4.17 & .874 & .759 & 17.94 & \\
\hline 23 & PR 65-218 & 10.95 & 35.45 & 3.81 & .798 & .693 & 17.14 & \\
\hline 24 & PR 64-211 & 10.72 & 35.70 & 3.64 & .763 & .663 & 17.28 & \\
\hline 25 & PR 65-229 & 10.91 & 32.79 & 3.53 & .740 & .642 & 16.40 & \\
\hline
\end{tabular}


second ratoon crop (table $3 \mathrm{C}$ ), it is evident that overall yield differences between varieties was minimized.

A combined statistical analysis of the data from the plant crop and the two ratoon crops (table 3 D) reveals that PR 1141 was the best variety under the conditions prevailing at Las Claras. It produced the best quality juice, i.e., $12.46 \%$ sucrose in the combined analysis of the three crops with a high value of 13.22 for the first ratoon (table $3 \mathrm{~B}$ ) and 11.84 for the plant crop (table $3 \mathrm{~A}$ ). These values were the highest obtained throughout the experimcntal cycle. PR 980 produced more cane tonnage than any other variety for the three crops but was relatively low in sucrose content.

Among the leading varieties, PR 1141 appears more desirable because of its consistently high sucrose content, relatively high tonnage and good agronomic characteristics. Even when PR 980 yielded 6 tons more cane than PR 1141, the sugar yields per acre of PR 1141 were higher. Still more relevant, PR 1141 is an erect cane suitable for mechanical harvesting. PR 62-285 (I) and PR 65-325 produced good tonnage and good quality juices but they exhibited undesirable characteristics for mechanized harvesting. PR 62-258 is also promising for the region, because of the high sucrose content and the semi-erect growth. In general, fiber content under Las Claras conditions seemed higher than usual, varying from 14.55 to $18.30 \%$ (table 3 D). The Sabana Seca compact subsoil in this area could limit available water supplying power, thus inducing stress in the cane plant. This condition could have been a factor in the increased fiber content.

\section{CONSEJO FARM SITE}

Performance data on the plant crop of the 28 varieties included in this experiment is given in table $4 \mathrm{~A}$. Data on tonnage only was obtained. Due to difficulties in the laboratory, juices did not clarify and sucrose contents of the juice and of the cane could not be determined. In terms of cane tonnage, most of the varieties performed very well, including PR 980 and PR 1028 both of which were used as check varieties.

Data on cane and sugar produced by the first ratoon crop is given in table 4 B. Selección Sóller, PR 65-2523, PR 1117, PR 1048, CB 49260, PR 1152, PR 980 and PR 62-469 all yielded over 4 tons/acre of sugar. Among them, PR 1152, and PR 62-469 seem to be very promising with high quality juices (12.73 and $12.69 \%$, sucrose respectively). These two varieties also have desirable growth habits.

As only cane tonnage production data are available for the second ratoon, the data are not included in the evaluation. 
TABLE 4.-Mean yields of 28 sugarcane varieties in the experimental field at Consejo Farm

\begin{tabular}{|c|c|c|c|c|c|c|c|c|}
\hline \multirow{2}{*}{ Rank } & \multirow{2}{*}{ Variety } & \multirow{2}{*}{$\begin{array}{l}\text { Sucrose } \\
\text { Content }\end{array}$} & \multirow{2}{*}{$\mathrm{TCA}^{1}$} & \multirow{2}{*}{$\mathrm{TSA}^{2}$} & \multicolumn{2}{|c|}{$\begin{array}{l}\text { Relative value on TSA as } \\
\text { compared to checks }\end{array}$} & \multirow{2}{*}{$\begin{array}{l}\text { Fiber } \\
\text { content }\end{array}$} & \multirow{2}{*}{$\begin{array}{c}\text { Outyielded } \\
\text { varieties } \\
\text { in TSA } \\
(\mathrm{P}=0.05)\end{array}$} \\
\hline & & & & & PR980 & PR1028 & & \\
\hline & & $\%$ & & & & & $\%$ & \\
\hline \multicolumn{9}{|c|}{ A. Plant cane crop 1972} \\
\hline 1 & PR 1048 & & 61.9 & & & & & \\
\hline 2 & PR 1117 & & 61.7 & & & & & \\
\hline 3 & PR 1152 & & 61.6 & & & & & \\
\hline 4 & PR 62-285(I) & & 60.8 & & & & & \\
\hline 5 & PR 1028 & & 57.8 & & & & & \\
\hline 6 & PR 980 & & 56.6 & & & & & \\
\hline 7 & PR $65-2523$ & & 55.7 & & & & & \\
\hline 8 & PR 1002 & & 55.7 & & & & & \\
\hline 9 & PR 65-292 & & 54.7 & & & & & \\
\hline 10 & PR $62-469$ & & 54.1 & & & & & \\
\hline 11 & PR 63-227 & & 53.7 & & & & & \\
\hline 12 & PR 64-2397 & & 52.5 & & & & & \\
\hline 13 & Sel Soller & & 51.4 & & & & & \\
\hline 14 & Q 68 & & 51.2 & & & & & \\
\hline 15 & PR $65-2507$ & & 49.9 & & & & & \\
\hline 16 & PR 65-1422 & & 48.7 & & & & & \\
\hline 17 & PR 1141 & & 48.5 & & & & & \\
\hline 18 & PR 62-626 & & 48.4 & & & & & \\
\hline 19 & PR 1140 & & 47.8 & & & & & \\
\hline 20 & C B $49-260$ & & 47.0 & & & & & \\
\hline 21 & PR 1248 & & 46.3 & & & & & \\
\hline 22 & PR 1175 & & 44.2 & & & & & \\
\hline 23 & CP $52-43$ & & 42.2 & & & & & \\
\hline 24 & PR $62-46$ & & 41.2 & & & & & \\
\hline 25 & PR 62-258 & & 40.9 & & & & & \\
\hline 26 & Sel Manatí & & 40.4 & & & & & \\
\hline
\end{tabular}




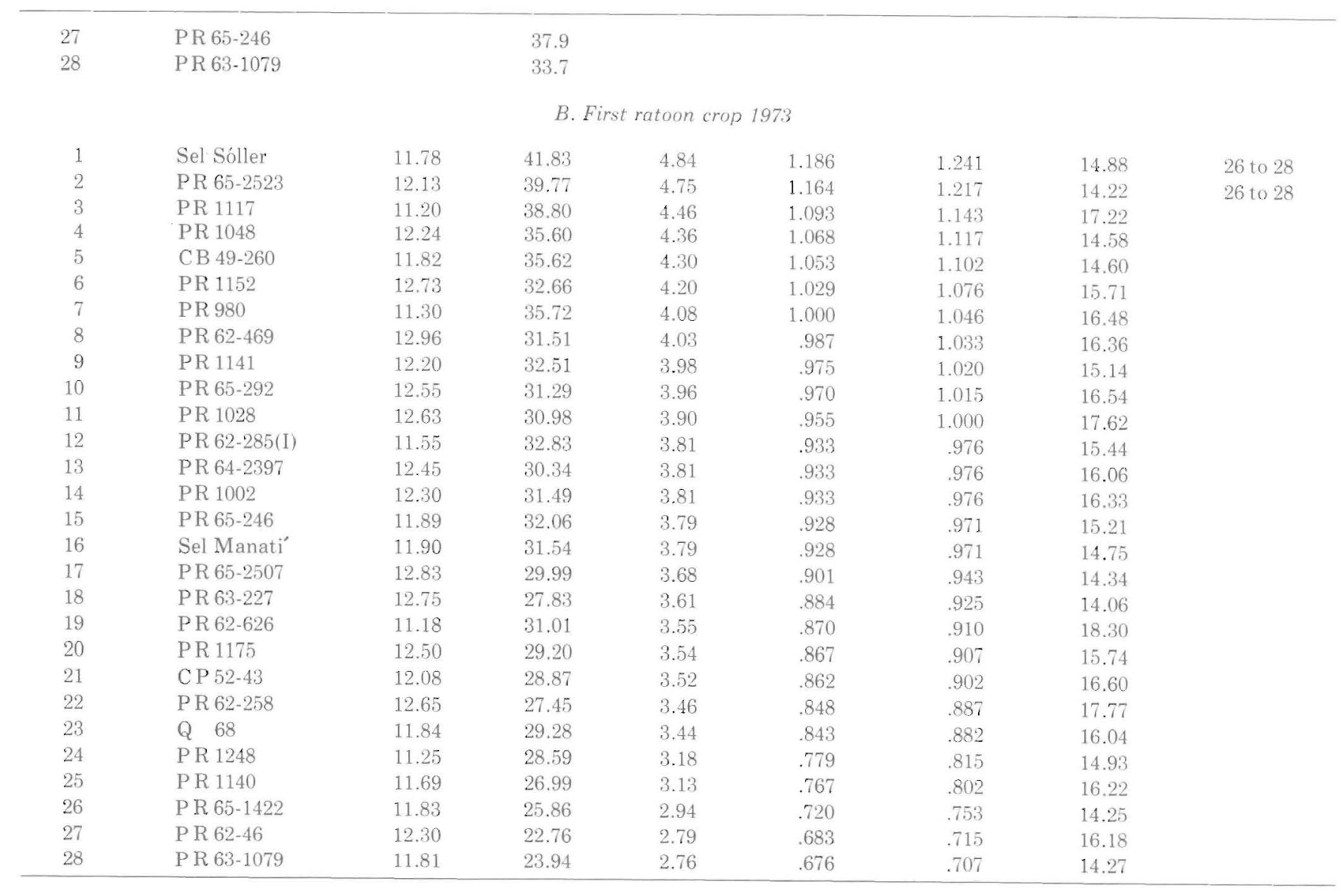


Table 4.-Continued

\begin{tabular}{|c|c|c|c|c|c|c|c|c|}
\hline \multirow{2}{*}{ Rank } & \multirow{2}{*}{ Variety } & \multirow{2}{*}{$\begin{array}{l}\text { Sucrose } \\
\text { Content }\end{array}$} & \multirow{2}{*}{ TÇA' } & \multirow{2}{*}{$\mathrm{TSA}^{2}$} & \multicolumn{2}{|c|}{$\begin{array}{l}\text { Relative value on TSA as } \\
\text { compared to checks }\end{array}$} & \multirow{2}{*}{$\begin{array}{l}\text { Fiber } \\
\text { content }\end{array}$} & \multirow{2}{*}{$\begin{array}{c}\text { Outyielded } \\
\text { varieties } \\
\text { in TSA } \\
(\mathrm{P}=0.05)\end{array}$} \\
\hline & & & & & PR 980 & PR 1028 & & \\
\hline & & $\%$ & & & & & $\%$ & \\
\hline \multicolumn{9}{|c|}{ C. Second ratoon crop 1974} \\
\hline 1 & PR 1141 & & 37.40 & & & & & \\
\hline 2 & Sel Sóller & & 35.97 & & & & & \\
\hline 3 & PR 1152 & & 35.50 & & & & & \\
\hline 4 & CB 49-260 & & 35.10 & & & & & \\
\hline 5 & PR 1002 & & 34.83 & & & & & \\
\hline 6 & PR 1117 & & 34.27 & & & & & \\
\hline 7 & PR 980 & & 32.72 & & & & & \\
\hline 8 & PR 62-258 & & 31.97 & & & & & \\
\hline 9 & PR 62-285(I) & & 31.93 & & & & & \\
\hline 10 & PR 1048 & & 31.18 & & & & & \\
\hline 11 & PR 65-2523 & & 30.82 & & & & & \\
\hline 12 & PR 65-2507 & & 30.70 & & & & & \\
\hline 13 & PR 1028 & & 29.97 & & & & & \\
\hline 14 & PR 1140 & & 29.42 & & & & & \\
\hline 15 & PR 62-626 & & 29.36 & & & & & \\
\hline 16 & PR 1248 & & 28.58 & & & & & \\
\hline 17 & PR 65-292 & & 28.19 & & & & & \\
\hline 18 & PR 64-2397 & & 27.87 & & & & & \\
\hline 19 & PR 63-227 & & 27.21 & & & & & \\
\hline 20 & PR 62-469 & & 26.16 & & & & & \\
\hline 21 & CP $52-43$ & & 25.14 & & & & & \\
\hline 22 & Sel Manati' & & 24.78 & & & & & \\
\hline 23 & PR 62-46 & & 24.50 & & & & & \\
\hline 24 & Q 68 & & 24.06 & & & & & \\
\hline 25 & PR 1175 & & 23.45 & & & & & \\
\hline 26 & PR 63-1079 & & 23.08 & & & & & \\
\hline 27 & PR 65-1422 & & 22.54 & & & & & \\
\hline 28 & PR 65-246 & & 21.57 & & & & & \\
\hline
\end{tabular}




\section{RESUMEN}

En los llanos costaneros de la zona norte húmeda de Puerto Rico, se seleccionaron tres sitios para evaluar el comportamiento de 57 nuevas variedades de caña de azúcar. Los experimentos se ubicaron en la finca San Francisco (carretera de Areibo a Utuado), en la finca Las Claras, cerca del molino azucarero Cambalache y en la finca Consejo, Barrio Bajadero, en Arecibo. Los suelos donde se ubicaron los experimentos se clasifican como Coloso, Sabana Seca y Toa, respectivamente. Estos son suelos típicos de los llanos cos. taneros de la zona norte dedicados al cultivo de la caña de azúcar en Puerto Rico.

Una siembra de gran cultura y dos retoños se cosecharon en cade uno de los experimentos. Se recopiló información sobre germinación, ahijamiento, madurez, hábito de crecimiento, rendimiento y tonelaje de caña y azúcar producidos por acre. Los valores obtenidos con respecto a rendimiento y producción de caña y de azúcar por acre se analizaron estadisticamente por cosecha. También, donde se justificó, se sometieron a análisis estadısticos combinado los datos de la plantilla y los retoños.

En San Francisco, las variedades nuevas sobresalientes, fueron la PR 64-2548, PR 1152 , PR 65-325 y la PR 1242. Estas produjeron más azúcar que las demás incluídas en el experimento. Tomando en consideración el hábito de crecimiento y el rendimiento, las variedades PR 1152 y PR 64-2705 parecen ser las más aceptables para la zona.

En la finca Las Claras, entre las nueve variedades superiores, las PR 114 I y PR 62-258 demuestran ser las más prometedoras, ya que producen rendimientos aceptables, tienen buenas características agronómicas y pueden cosecharse mecánicamente.

En la finca Consejo la PR 1152 parece ser la mejor, pues alcanzó un rendimiento superior y sobrepasó las 40 toneladas de caña por acre. También puede cosecharse mecánicamente.

La variedad PR 980 produjo un tonelaje por acre superior a la PR 1152, pero fue inferior en contenido de sacarosa. La calidad inferior de los jugos de la PR 980 y el crecimiento reclinado nos indica que esta variedad no es recomendable para los llanos costaneros de la zona húmeda del norte.

\section{LITERATURE CITED}

1. González Molina, Carlos et al., Comportamiento agronómico de nuevas variedades de caña de azúcar en Puerto Rico, Est. Exp. Agr. Univ. P.R. Publicación 81, 1970.

2. Pringle, George C., Farm value of agricultural commodities by rank, Puerto Rico, 1973-74, Mimeogr. Rep. Agr. Exp. Sta. Univ. P.R., 1974.

3. Soil Conservation Service, USDA, Soil series of United States, Puerto Rico and the Virgin Islands: Their taxonomic classification, 1972.

4. Troche, Carlos, Información de Prensa Núm. 1120, Servicio de Estabilización y Conservación Agricola, Area del Caribe, Departamento de Agricultura de los Estados Unidos, 1974. 